\title{
Charmonium and $\mathrm{e}^{+} \mathrm{e}^{-}$photoproduction in ultra peripheral $\mathrm{Pb}-\mathrm{Pb}$ collisions at $\sqrt{ } \mathrm{s}_{\mathrm{nn}}=2.76 \mathrm{TeV}$
}

\author{
Eugenio Scapparone on behalf of the ALICE Collaboration ${ }^{1}$ \\ INFN-Bologna \\ Via Irnerio 46, 40126, Bologna (Italy) \\ E-mail: eugenio.scapparone@bo.infn.it
}

Ultra-relativistic heavy ions generate strong electromagnetic fields which offer the possibility to study $\gamma \gamma$ and $\gamma$-nucleus interactions at the LHC in the so called ultra-peripheral collisions(UPC). The $\mathrm{J} / \psi$ photoproduction in UPC events is sensitive to the gluon distribution of the interacting nuclei, providing information on the nuclear gluon shadowing at Bjorken- $x$ ranging from $10^{-2}$ to $10^{-3}$. Here we report on ALICE results of $\mathrm{J} / \psi$ photoproduction measured in $\mathrm{Pb}-\mathrm{Pb}$ collisions at $\sqrt{\mathrm{s}_{\mathrm{NN}}}=2.76 \mathrm{TeV}$ where the $\mathrm{J} / \psi$ has been measured in the $\mu \mu$ decay channel with the ALICE Muon Spectrometer in the rapidity range $-3.6<\mathrm{y}<-2.6$; at midrapidity $(|\mathrm{y}|<0.9)$ both the $\mathrm{J} / \psi$ leptonic decay channels $\left(\mathrm{e}^{+} \mathrm{e}^{-}, \mu^{+} \mu^{-}\right)$were used. The cross sections are compared with the predictions provided by several Monte Carlo simulations. In addition the cross section for the $\gamma \gamma \rightarrow \mathrm{e}^{+} \mathrm{e}^{-}$process was studied and compared with the theoretical model expectations.

XXI International Workshop on Deep-Inelastic Scattering and Related Subjects

April 22-26, 2013

Marseilles, France

${ }^{1}$ Speaker 


\section{Introduction}

Collisions between heavy-ions at the LHC can be used to study particle production in photonuclear and two-photon interactions [1]. These interactions may occur in ultra-peripheral collisions (UPC), where the impact parameter is larger than twice the $\mathrm{Pb}$ radius. The background from hadronic processes in this category of events is negligible. Exclusive photoproduction of vector mesons in heavy-ion interactions has attracted an increased theoretical interest in recent years $[2,3,4,5]$. The first results from the Brookhaven Relativistic Heavy-Ion Collider showed the experimental feasibility of these studies, and the increased energy at the LHC leads to significantly higher cross sections for heavy vector mesons. The higher energy reflects in a decrease of the probed parton Bjorken- $x$. Exclusive photonuclear production of vector mesons implies that there is no net color transfer between the photon and the nucleus. The events considered here are thus characterized by a single $\mathrm{J} / \psi$ meson but no other particles being produced. The cross section for this process is proportional to the nuclear gluon distribution function, shedding light on the low Bjorken- $x$ region, where the nuclear gluon shadowing is poorly known. In this talk, results on coherent photoproduction of $\mathrm{J} / \psi$ vector mesons and two-photon production of electron-pairs measured by the ALICE Collaboration at the LHC are presented $[8,9]$. The $\mathrm{J} / \psi$ is studied through its di-muon decay at forward rapidity and both di-muon and di-electron decay at mid-rapidity. Another process with similar topology is two-photon production of di-lepton pairs, studied at mid-rapidity in the di-electron channel. Two-photon interactions and coherent photoproduction are both associated with a low $p_{T}(<100$ $\mathrm{MeV} / \mathrm{c}$ ) of the final state. To take into account also the finite detector resolution, a $p_{T}$ cut is used to define coherent interactions in the di-muon(di-electron) channel for the present analysis.

\section{The ALICE Experiment}

The ALICE detector consists of a central barrel placed inside a large solenoid magnet $(0.5 \mathrm{~T})$ covering the pseudorapidity range $|\eta|<0.9$, a muon spectrometer covering the range $-4.0<\eta<-2.5$, and a set of smaller detectors at forward rapidity. The forward rapidity analysis is based on the muon spectrometer. In addition, the VZERO counters and Zero-Degree Calorimeters (ZDC) are used for triggering UPC and rejecting the contribution from hadronic interactions. The ALICE muon spectrometer consists of five tracking stations containing two planes of cathode pad multi-wire proportional chambers. A ten interaction length thick absorber is placed between the primary vertex and the first tracking station. The third (middle) tracking station is situated inside a dipole magnet. The VZERO counters are arrays of scintillator tiles situated on either side of the primary vertex at pseudorapidities $2.8<\eta<5.1$ (VZERO-A, on the opposite side of the muon spectrometer) and $-3.7<\eta<-1.7$ (VZERO-C, on the same side as the muon spectrometer). The ZDCs are hadronic calorimeters located at $116 \mathrm{~m}$ on either side of the interaction point.They detect neutrons in the very forward region $(/ \eta />8.8)$. The analysis at mid-rapidity uses the barrel detectors (SPD, TPC and TOF), the VZERO counters and the ZDC calorimeters. More details on the barrel detectors used for this analysis can be found in [9].

\section{$3 \mathrm{~J} / \psi$ data analysis \\ 3.1 Forward rapidity}

The analysis at forward rapidity is based on a sample of $3 \cdot 10^{6}$ events collected with a ultraperipheral collisions dedicated trigger (FUPC) during the $2011 \mathrm{~Pb}-\mathrm{Pb}$ run. The purpose of the FUPC trigger was to select two muons in an otherwise empty detector. It is based on three requirements: a single muon trigger above a $1 \mathrm{GeV} / \mathrm{c} \mathrm{p}_{\mathrm{T}}$ threshold, at least one hit in VZERO-C, 
no hits in VZERO-A. The offline event selection was done in such a way as to maximize the yield of exclusively produced muon pairs from $\mathrm{J} / \psi$ decay and two-photon interactions, while minimizing the background from hadronic collisions and beam gas interactions. Only events with two opposite charged tracks in the muon spectrometer were considered. The analysis was restricted to $\mathrm{J} / \psi$ rapidities between $-3.6<y<2.6$ and muon pseudorapidities between $-3.7<$ $\eta_{1,2}<-2.5$ to match the acceptance of the VZERO-C and avoid the edges of the spectrometer. A $p_{T}$ dependent cut on the distance of closest approach of the tracks from the primary vertex position in the transverse plane was applied, and at least one of the muon track candidates had to match a trigger track above the $\mathrm{GeV} / \mathrm{c}$ threshold. More details about the analysis cuts are available in [8]. Exclusive photoproduction of vector mesons normally leaves the nuclei intact. But the strong fields associated with heavy-ions at high energies make the exchange of multiple photons possible. These additional photons have low energy but may lead to nuclear break up, followed by emission of one or a few neutrons in the forward region. The energy deposit in the $\mathrm{ZDCs}$ was required to be less than $6 \mathrm{TeV}$. The invariant mass distribution for the di-muons surviving the analysis cuts are shown in Figure 1 (left). The final sample contained $117 \mathrm{~J} / \psi$ candidates in the invariant mass range $2.8<m_{i n v}<3.4 \mathrm{GeV} / \mathrm{c}^{2}$. The number of $\mathrm{J} / \psi \mathrm{s}$ was extracted by fitting the invariant mass distribution to a Crystal Ball function, for the signal, and an exponential, for the background from two-photon interactions. This gave an extracted number of J/ $\psi \mathrm{s}, N_{\text {yield }}=96 \pm 12$ (stat) \pm 6 (syst). The systematic error on the yield was obtained by varying the Crystal Ball tail parameters. The transverse momentum distribution of $\mathrm{J} / \psi$ candidates is shown in fig 1 (right). The histograms show the expected contribution from coherent $\mathrm{J} / \psi$ production, incoherent $\mathrm{J} / \psi$ production, $\mathrm{J} / \psi$ from the decay $\psi^{\prime} \rightarrow \mathrm{J} / \psi+\mathrm{X}$, and two-photon production of dimuon pairs. As can be seen, the signal region $p_{T}<0.3 \mathrm{GeV} / \mathrm{c}$ contains background contributions from incoherent $\mathrm{J} / \psi$ production as well as feed down from $\psi$ 'decay. These contributions can be expressed as fractions of the number of coherent $\mathrm{J} / \psi \mathrm{s}$. The method used to estimate these fractions and the associated uncertainties is explained in detail in [8]. The fraction $\mathrm{f}_{\mathrm{D}}$ of $\mathrm{J} / \psi \mathrm{s}$ from $\psi$ 'decay was obtained from the calculated $\psi$ ' photoproduction cross section from the models in [5] and [6]. The acceptance and efficiency for reconstructing a $\mathrm{J} / \psi$ was obtained by simulating the decay $\psi$ ' $\rightarrow \mathrm{J} / \psi+\mathrm{X}$ with Pythia and passing the events through the ALICE Geant detector simulation package. The result was $f_{D}$ $=0.11 \pm 0.06$. The fraction of incoherent events was similarly obtained from model calculations $[5,6]$ followed by simulations of the detector response. As a cross check, it was also obtained by fitting the $\mathrm{p}_{\mathrm{T}}$ distribution in fig. 1 to Monte Carlo templates representing the four contributions mentioned above. The relative normalization for coherent and incoherent production was left free in the fit, while the contribution from feed-down from photoproduced $\psi$ 'was constrained from the estimate $f_{D}=0.11 \pm 0.06$. The two-photon contribution was obtained from the fit of the di-muon continuum outside the $\mathrm{J} / \psi$ peak. This gave a result $\mathrm{f}_{\mathrm{I}}=0.12_{-0.04}^{+0.14}$. The contribution from hadronic $\mathrm{J} / \psi$ production was estimated from the measured yield above $\mathrm{p}_{\mathrm{T}}>1 \mathrm{GeV} / \mathrm{c}$, where the contribution from photoproduction is negligible, and was found to be smaller than $1 \%$ in the $\mathrm{p}_{\mathrm{T}}<0.3 \mathrm{GeV} / \mathrm{c}$ region considered here. The number of coherent $\mathrm{J} / \psi \mathrm{s}$ is related to the extracted yield by

$$
\mathrm{N}_{\mathrm{J} / \psi}^{\mathrm{coh}}=\mathrm{N}_{\mathrm{J} / \psi}^{\mathrm{yield}} /\left(1+\mathrm{f}_{\mathrm{I}}+\mathrm{f}_{\mathrm{D}}\right)
$$

The resulting number of coherent $\mathrm{J} / \psi$ s is $78 \pm 10$ (stat) ${ }_{-11}^{+7}$ (syst). During the $2011 \mathrm{~Pb}-\mathrm{Pb}$ run, the 

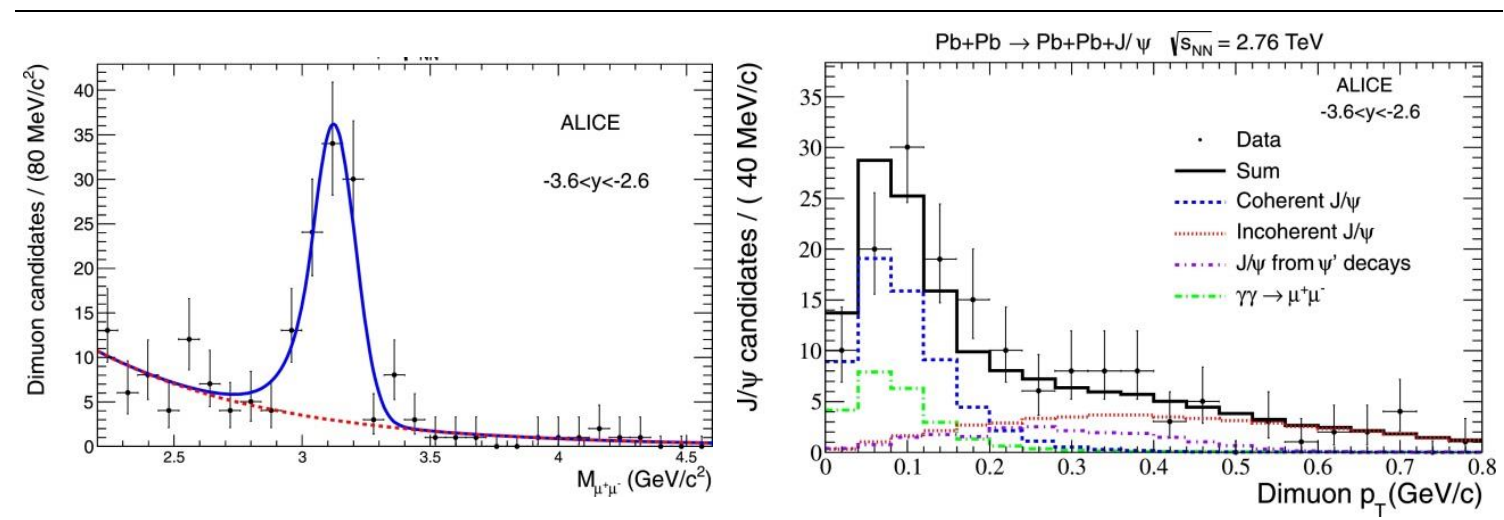

Fig. 1 Ultra-peripheral collisions at ${\sqrt{\mathrm{s}_{\mathrm{NN}}}}=2.76 \mathrm{TeV}$ and $-3.6<\mathrm{y}<-2.6:$ di-muon invariant mass distribution (left) and $\mathrm{p}_{\mathrm{T}}$ distribution(right).

VZERO detector had a threshold corresponding to an energy deposit above that from a minimum ionizing particle. This made it difficult to accurately simulate the VZERO-C trigger efficiency for low multiplicity events. To avoid these uncertainties, the $\mathrm{J} / \psi$ cross section was obtained by using the number of reconstructed $\gamma \gamma \rightarrow \mu^{+} \mu^{-}$events. The $\mathrm{J} / \psi$ cross section can then be written in a way that is independent of the trigger efficiency and the integrated luminosity (see ref. [8] for more details),

$$
\mathrm{d} \sigma_{\mathrm{J} / \psi /} / \mathrm{dy}=\mathrm{N}_{\mathrm{J} / \psi}^{\mathrm{coh}} \cdot(\mathrm{Acc} \cdot \varepsilon) \gamma \cdot \sigma_{\gamma /} /\left(\left(\mathrm{N}_{\gamma \gamma} \cdot \Delta \mathrm{y} \cdot \mathrm{BR}\left(\mathrm{J} / \psi \rightarrow \mu^{+} \mu^{-}\right) \cdot(\mathrm{Acc} \cdot \varepsilon) \mathrm{J} / \psi\right)\right.
$$

Here, $(\text { Acc } \cdot \varepsilon)_{\gamma}$ and $($ Acc $\varepsilon) \mathrm{J} / \psi$ are the acceptance and efficiency of the muon spectrometer for two-photon and coherent $\mathrm{J} / \psi$ events, respectively. $\mathrm{BR}\left(\mathrm{J} / \psi \rightarrow \mu^{+} \mu^{-}\right)$is the branching ratio for the dimuon decay of the $\mathrm{J} / \psi$ and $\Delta y=1.0$ is the rapidity interval. $\mathrm{N}_{\gamma \gamma}$ was obtained by counting the number of events in the invariant mass intervals $2.2<m_{\text {inv }}<2.6 \mathrm{GeV} / \mathrm{c}^{2}$ and $3.5<m_{\text {inv }}<6.0$ $\mathrm{GeV} / \mathrm{c}^{2}$ to avoid contamination from the $\mathrm{J} / \psi$ peak. To determine $\sigma_{r \prime}$ for a di-muon within $3.6<y<2.6$ and each muon satisfying $-3.7<\eta_{1,2}<-2.5$, STARLIGHT[11] was used. The number of events found in each interval is 43 and 15 , for the low and high mass interval, respectively. This together with the cross sections, $\sigma_{w}=13.7 \mu \mathrm{b}\left(\right.$ low $\left.m_{i n v}\right)$ and $3.7 \mu \mathrm{b}\left(\right.$ high $m_{\text {inv }}$ ), gave a total cross section for $-3.6<y<-2.6$

$$
\mathrm{d} \sigma^{\mathrm{coh}} / \mathrm{dy}(\mathrm{J} / \psi)=1.00 \pm 0.18(\text { stat }){ }_{-0.26}^{+0.24} \text { (syst) mb. }
$$

The systematic error is dominated by the uncertainty in the two-photon cross section. Although this is a standard QED process, the fact that the coupling to the nuclei is not small ( $Z \sqrt{ } \alpha$ rather than $\sqrt{ } \alpha$ ) and that the photon emitting nuclei are extended objects with an internal structure introduces a significant uncertainty in the cross section. Based on calculations [5] and constraints from data from RHIC, this uncertainty is estimated to be $20 \%$. Other contributions to the systematic error are the signal extraction and the reconstruction efficiency [8].

\subsection{Mid-rapidity analysis}

The measurement of $\mathrm{J} / \psi$ production in UPC at mid-rapidity was obtained using a sample of $6.5 \cdot 10^{6}$ events collected collected during the $2011 \mathrm{~Pb}-\mathrm{Pb}$ run. The trigger required a number of fired pad-OR in the time-of-flight detector (TOF) in the range $2 \leq \mathrm{N}^{\mathrm{on}} \leq 6$, with at least two of them back-to-back, two hits in the silicon pixel detector (SPD) and vetoes on VZERO counters at forward and backward rapidity. Track reconstruction was performed with the time projection chamber (TPC) and the silicon tracking system in about two pseudorapidity units. Events with 
only two opposite sign good-quality tracks coming from a reconstructed primary vertex have been selected. $\mathrm{J} / \psi$ signal was extracted both in dielectron and dimuon channels, which were separated by the energy deposition in TPC. A sample was enriched with coherent events by applying a cut $\mathrm{p}_{\mathrm{T}}<200 \mathrm{MeV} / \mathrm{c}$ for di-muons $\left(\mathrm{p}_{\mathrm{T}}<300 \mathrm{MeV} / \mathrm{c}\right.$ for di-electrons) and requiring a $\mathrm{ZDC}$ energy deposit $\mathrm{E}<6 \mathrm{TeV}$. The $\mathrm{J} / \psi$ yield was obtained by fitting the di-lepton invariant mass spectrum with an exponential function to describe the underlying continuum, and a Crystal Ball function to extract the $\mathrm{J} / \psi$ signal. As an example fig. 1 (left) shows the invariant mass distribution for the di-electron sample. The number of coherent $\mathrm{J} / \psi$ was then obtained using formula (1), giving $255 \pm 16$ (stat) ${ }_{-0.13}^{+0.14}$ (sys) for di-muon channel and $212 \pm 32$ (stat) ${ }_{-0.13}^{+0.14}$ (sys) for di-electron channel respectively. The acceptance and the efficiency of $\mathrm{J} / \psi$ reconstruction were calculated using a large sample of coherent $J / \psi$ events generated by STARLIGHT[11]. The $f_{D}$ and $f_{I}$ fractions were obtained using the same prescription described in the forward analysis. Cross section normalization was performed with respect to the integrated luminosity which was measured using a trigger for the most central hadronic $\mathrm{Pb}-\mathrm{Pb}$ collisions and corrected
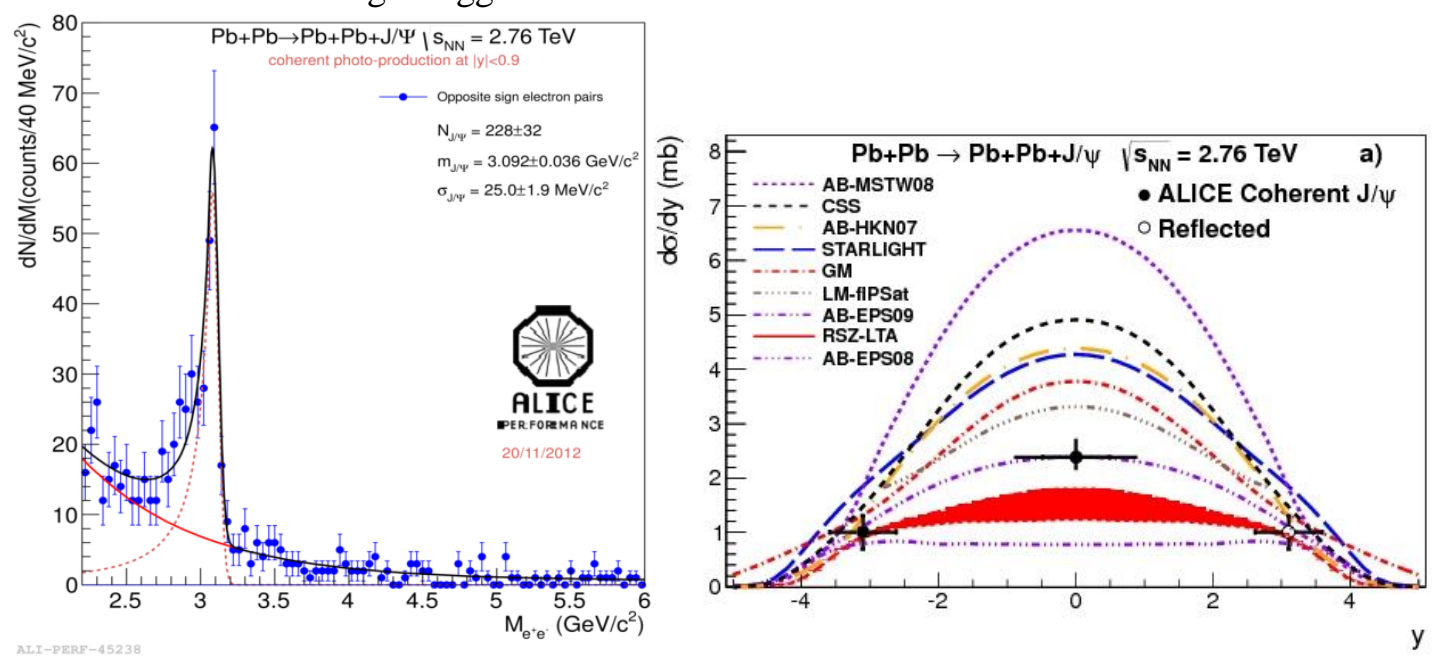

Fig. 2 Di-electron invariant mass distribution for ultra-peripheral collisions at ${\sqrt{\mathrm{S}_{\mathrm{NN}}}}=2.76 \mathrm{TeV}$ (left) and measured $\mathrm{J} / \psi$ differential cross section for coherent events (right).

for the UPC trigger live time. Results have been recently published in [9], giving for $|y|<0.9$

$$
\mathrm{d} \sigma^{\mathrm{coh}} / \mathrm{d} y(\mathrm{~J} / \psi)=2.38_{-0.24}^{+0.34}(\text { stat+syst }) \mathrm{mb} .
$$

\subsection{Comparison with models}

The measured cross section can be compared with the models mentioned earlier [2,3,4,5]. The models by Lappi and Mäntysaari [3], Cise et al. [4], and Goncalves and Machado [5] are based on the Color Dipole Model (CDM). The model by Klein and Nystrand[7], which is incorporated in the STARLIGHT, uses data from exclusive vector meson production at HERA as input to a Glauber calculation of the cross section for nuclear targets. The models by Rebyakova et al. [2] and Adeluyi and Bertulani [6], calculate the cross section directly from the nuclear gluon distribution with the forward scattering amplitude being proportional to the gluon distribution squared. Rebyakova et al. calculate the modifications to the nuclear gluon distribution in the leading twist approximation, while Adeluyi and Bertulani use standard parameterizations (HKN07, EPS09, and EPS08). Adeluyi and Bertulani also calculate the cross section by scaling the $\gamma+p \rightarrow \mathrm{J} / \psi+p$ cross section with the number of nucleons assuming no nuclear effects (MSTW08). The results are shown in fig. 2(right). One can see that models which are based on the color dipole model generally give a higher cross section than those which calculate the cross 
section directly from the gluon distribution. This is true at the forward rapidities studied here, but is emphasized even more at mid-rapidity. The MSTW08 scaling of the cross section without nuclear effects and STARLIGHT deviate by a factor 2-3 from the measured value and are disfavored. Best agreement is found for models which include nuclear gluon shadowing consistent with the EPS09 parameterizations. The calculation by Lappi and Mäntysaari is in the same range as the other models using the color dipole model.

\section{Two-photon cross section}

The cross section for $\gamma \gamma \rightarrow \mathrm{e}^{+} \mathrm{e}^{-}$can be written as

$$
\sigma_{\gamma \gamma}=\mathrm{N}_{\gamma \gamma} /\left((\operatorname{Acc} \cdot \varepsilon)_{\gamma \gamma} \cdot \mathscr{L}\right),
$$

where $\mathrm{N}_{\gamma /}$ was obtained by fitting the continuum in the invariant mass intervals $2.2<\mathrm{m}_{\text {inv }}<2.6 \mathrm{GeV} / \mathrm{c}^{2}$ and $3.7<\mathrm{m}_{\text {inv }}<10 \mathrm{GeV} / \mathrm{c}^{2}$, to avoid contamination from the $\mathrm{J} / \psi$ peak. In this analysis the integrated luminosity used was $\mathscr{L}=21.7_{-1.1}^{+0.7}(s y s) \mu \mathrm{b}^{-1}$. The cross section for the process $\gamma \gamma \rightarrow \mu^{+} \mu^{-}$was not studied due to a possible contamination from pions in the di-muon sample, suggested by the presence of LS events. The cross section for di-electron invariant mass was computed for a di-electron rapidity in the interval $|y|<0.9$, and requiring $\left|\eta_{1,2}\right|<0.9$ for each lepton. The data cuts applied to the Monte Carlo sample are the same as those applied in the analysis described above, resulting in (Acc $\cdot \varepsilon)_{\gamma \gamma}=5.6 \%$ for $2.2<\mathrm{m}_{\mathrm{inv}}<2.6 \mathrm{GeV} / \mathrm{c}^{2}$ and $(\text { Acc } \cdot \varepsilon)_{\gamma \gamma}=4.73 \%$ for $3.7<\mathrm{m}_{\text {inv }}<10 \mathrm{GeV} / \mathrm{c}^{2}$. As a result we obtain $\sigma_{\gamma \gamma}=154 \pm 11(\mathrm{sta}){ }_{-11}^{+17}(\mathrm{sys}) \mu \mathrm{b}$ for the lower invariant mass interval and $\sigma_{\gamma \gamma}=91 \pm 10(\text { sta })_{-8}^{+11}($ sys $) \mu \mathrm{b}$ for the higher one, to be compared with $\sigma_{\gamma \gamma}=128 \mu \mathrm{b}$ and $\sigma_{\gamma \gamma}=77 \mu \mathrm{b}$ given by STARLIGHT. The measured values for the $\gamma \gamma$ cross sections are $20 \%$ above but compatible within 1.0 and 1.5 sigma with the STARLIGHT prediction for the low and high invariant mass intervals, respectively, if the statistical and systematic errors are added in quadrature. This result provides constraints on calculations that include terms of higher orders in $\alpha_{\mathrm{em}}$, predicting a cross section up to a $30 \%$ lower[12].

\section{References}

[1] A. J. Baltz et al., Phys. Rep. 458 (2008) 1.

[2] V. Rebjakova et al., Phys. Lett. B 710 (2012) 647.

[3] T. Lappi and H. Mantysaari, arXiv:1301.4095[nucl-th]

[4] A. Cizek et al., Phys. Rev. C 86 (2012) 014905.

[5] V. P. Goncalves and M.V.T. Machado, Phys. Rev. C 84 (2011) 011902.

[6] A. Adeluyi and Bertulani, Phys. Rev. C 85 (2012) 044942.

[7] S. R: Klein and J. Nystrand, Phys. Rev. C 60 (1999) 014903.

[8] B. Abelev et al. (ALICE Collaboration) Phys. Lett. B 718 (2013) 1273.

[9] B. Abelev et al. (ALICE Collaboration), arXiv:1035.1467.

[10]K. Aamodte et al. (ALICE Collaboration), JINST 3 (2008) S08002.

[11]STARLIGHT website, http://starlight.hepforge.org

[12]A. J. Baltz, Phys. Rev. C 80 (2009) 034901. 